\title{
Coherence and clock shifts in ultracold Fermi gases with resonant interactions
}

\author{
Gordon Baym, ${ }^{a, b}$ C. J. Pethick, ${ }^{b, c}$ Zhenhua Yu, ${ }^{a}$ and Martin W. Zwierlein ${ }^{d, e}$ \\ ${ }^{a}$ Department of Physics, University of Illinois, 1110 W. Green Street, Urbana, IL 61801 \\ ${ }^{b}$ The Niels Bohr Institute, Blegdamsvej 17, DK-2100 Copenhagen Ø, Denmark \\ ${ }^{c}$ NORDITA, Roslagstullsbacken 23, SE-10691 Stockholm, Sweden \\ ${ }^{d}$ MIT-Harvard Center for Ultracold Atoms, Research Laboratory for Electronics, Department of Physics, \\ Massachusetts Institute of Technology, Cambridge, MA 02139 \\ ${ }^{e}$ Institut für Physik, Johannes Gutenberg-Universität, 55099 Mainz, Germany
}

(Dated: August 26, 2021)

\begin{abstract}
Using arguments based on sum rules, we derive a general result for the average shifts of rf lines in Fermi gases in terms of interatomic interaction strengths and two-particle correlation functions. We show that, near an interaction resonance, shifts vary inversely with the atomic scattering length, rather than linearly as in dilute gases, thus accounting for the experimental observation that clock shifts remain finite at Feshbach resonances.
\end{abstract}

PACS numbers: 03.75.Hh, 05.30.Jp, 67.40.Db, 67.40.Vs

Interatomic interactions limit the accuracy of atomic clocks, causing density-dependent clock shifts in radio frequency (rf) transitions. Similarly, such shifts play an important role in probing correlations in atomic gases [1], where, e.g., rf spectroscopy has been used to detect the presence of molecules and provide evidence for pairing gaps [2, 3, 4]. Surprisingly, experimentally observed clock shifts become small when interactions are resonantly enhanced [3, 5], a result we explain here, first by developing a general theory of the average clock shift and then showing that in the strongly interacting regime the shifts depend inversely on interatomic scattering lengths.

Single component spin-polarized Fermi gases do not experience clock shifts, since the rf coupling preserves polarization, forbidding s-wave interactions [5]. In addition, in mixtures of interacting fermions in two states $|1\rangle$ and $|2\rangle$, e.g., the lowest two hyperfine states of ${ }^{6} \mathrm{Li}$, clock shifts are absent for transitions $|1\rangle \rightarrow|2\rangle$, since the interaction energy is invariant under the rf field [1]. Interactions in two-state mixtures can be probed, rather, by driving transitions to an initially empty state, e.g., in ${ }^{6} \mathrm{Li}$, from $|2\rangle$ to the next hyperfine state $|3\rangle$.

$\mathrm{Rf}$ transitions in atoms are usually described in terms of coherent evolution of a two-level system undergoing Rabi oscillations, represented by rotations of an equivalent pseudospin on the Bloch sphere. In the present problem, the rf field rotates an atom in state $|2\rangle$ into a coherent superposition, $|2\rangle \rightarrow|\beta\rangle=\cos \theta|2\rangle+e^{-i \phi} \sin \theta|3\rangle$. (The angles $\phi$ and $\theta$ depend on time, the rf pulse power, and its detuning from resonance.) To the extent that interatomic interactions only shift the energy levels, but do not broaden the lines, the Bloch sphere picture is valid. However, strong interactions lead to an incoherent and irreversible evolution, and in the long-time, weak pulse regime the probability to find the system in a particular final state is given rather by Fermi's Golden Rule.

We base our discussion on linear response theory (in Ref. [5] fewer than $30 \%$ of the atoms are transferred by the rf pulse, with comparable numbers in Ref. [3]). We consider a spatially uniform Fermi gas with three internal states, and Hamiltonian $H=H_{0}+H_{\mathrm{s}}$, where $H_{0}=\sum_{i=1}^{3} \epsilon_{i} N_{i}, \epsilon_{i}$ is the hyperfine plus Zeeman energy of level $|i\rangle$, and $N_{i}$ is the total number of atoms in state $|i\rangle$. The system Hamiltonian is ( $\hbar=1$ throughout),

$$
\begin{array}{r}
H_{\mathrm{s}}=\int d^{3} r\left(\frac{1}{2 m} \nabla \psi_{i}^{\dagger}(\mathbf{r}) \cdot \nabla \psi_{i}(\mathbf{r})\right) \\
+\sum_{i<j} \int d^{3} r d^{3} r^{\prime} v_{i j}\left(\mathbf{r}-\mathbf{r}^{\prime}\right) \psi_{i}^{\dagger}(\mathbf{r}) \psi_{j}^{\dagger}\left(\mathbf{r}^{\prime}\right) \psi_{j}\left(\mathbf{r}^{\prime}\right) \psi_{i}(\mathbf{r})
\end{array}
$$

Here $v_{i j}\left(\mathbf{r}-\mathbf{r}^{\prime}\right)$ is the bare potential between two atoms in $|i\rangle$ and $|j\rangle$ (not a low-energy effective interaction).

The (essentially spatially uniform) rf field primarily couples the states $|2\rangle$ and $|3\rangle$; other internal transitions are far off-resonance. We therefore describe the rf coupling by $H_{\mathrm{rf}}=\mathcal{B}(t) Y$ with the pseudospin-flip operator

$$
Y=i \int d^{3} r\left(\psi_{3}^{\dagger}(\mathbf{r}) \psi_{2}(\mathbf{r})-\psi_{2}^{\dagger}(\mathbf{r}) \psi_{3}(\mathbf{r})\right),
$$

where $\mathcal{B}(t)=2 \omega_{\mathrm{R}} \cos \omega t$, with $\omega_{\mathrm{R}}$ the Rabi frequency.

We assume an initial many-body state $|12\rangle$ of energy $E_{12}$, containing atoms in states $|1\rangle$ and $|2\rangle$. The transition rate of atoms from state $|2\rangle$ to $|3\rangle, I(\omega)$, is

$$
I(\omega)=2 \pi \omega_{\mathrm{R}}^{2} \sum_{f}|\langle f|Y| 12\rangle|^{2} \delta\left(\omega+E_{12}-E_{f}\right) \equiv 2 \omega_{\mathrm{R}}^{2} \chi^{\prime \prime}(\omega),
$$

where the sum runs over all eigenstates $|f\rangle$. The mean frequency for the transition,

$$
\bar{\omega} \equiv \frac{\int_{-\infty}^{\infty} d \omega \omega \chi^{\prime \prime}(\omega)}{\int_{-\infty}^{\infty} d \omega \chi^{\prime \prime}(\omega)} \equiv \omega_{0}+\Omega_{\mathrm{c}},
$$

where $\omega_{0}=\epsilon_{3}-\epsilon_{2}$, and $\Omega_{\mathrm{c}}$ is the average clock shift. Using Eq. (3) we derive the f-sum rule for the first moment 
of the spectrum,

$$
\begin{aligned}
\int_{-\infty}^{\infty} \frac{d \omega}{\pi} \omega \chi^{\prime \prime}(\omega) & =\sum_{f}\left(E_{f}-E_{12}\right)|\langle f|Y| 12\rangle|^{2} \\
& =\frac{1}{2}\langle 12|[[Y, H], Y]| 12\rangle .
\end{aligned}
$$

For state $|3\rangle$ initially unoccupied,

$$
\begin{gathered}
\int_{-\infty}^{\infty} \frac{d \omega}{\pi} \chi^{\prime \prime}(\omega) \\
=\int d^{3} r d^{3} r^{\prime}\left\langle 12\left|\psi_{2}^{\dagger}(\mathbf{r}) \psi_{3}(\mathbf{r}) \psi_{3}^{\dagger}\left(\mathbf{r}^{\prime}\right) \psi_{2}\left(\mathbf{r}^{\prime}\right)\right| 12\right\rangle=N_{2},
\end{gathered}
$$

where $N_{2}$ is the number of atoms in state $|2\rangle$. We thus derive the simple result for the mean clock shift

$$
\Omega_{\mathrm{c}}=\frac{1}{2 N_{2}}\left\langle 12\left|\left[\left[Y, H_{\mathrm{s}}\right], Y\right]\right| 12\right\rangle .
$$

More generally, the expectation value becomes an ensemble average.

The average shift is simply the change in energy per 2 -atom required to rotate all $|2\rangle$ atoms in state $|12\rangle$ infinitesimally into state $|\beta\rangle$. This rotation transforms $|12\rangle$ into a state $|1 \beta\rangle=e^{i \theta Y}|12\rangle$ of $|1\rangle$ and $\beta$ atoms, with the same spatial many-particle wave function as $|12\rangle$. The energy difference of the two states is thus $\delta E=\langle 1 \beta|H| 1 \beta\rangle-\langle 12|H| 12\rangle \rightarrow \frac{1}{2} \theta^{2}\langle 12|[[Y, H], Y]| 12\rangle$ to second order in $\theta$. Since the number of atoms $\delta N$ transferred from $|2\rangle$ to $|3\rangle$ is $N_{2} \theta^{2}$, the change in system energy per atom is given by Eq. (7), a result valid both for superfluid and normal phases. The general expression for the clock shift in terms of the interactions is

$$
\Omega_{\mathrm{c}}=\frac{1}{n_{2}} \int d^{3} r\left[v_{13}(r)-v_{12}(r)\right]\left\langle\psi_{1}^{\dagger}(\mathbf{r}) \psi_{2}^{\dagger}(0) \psi_{2}(0) \psi_{1}(\mathbf{r})\right\rangle,
$$

where $n_{2}=N_{2} / V, V$ is the system volume, and $\langle\ldots\rangle$ denotes an ensemble average.

To calculate the clock shift explicitly, we assume for simplicity in the following that the interatomic potentials are short range contact interactions, each with a large momentum cutoff. We also assume that $n_{1}=n_{2}=n$. The bare couplings, $\bar{g}_{i j}$, are related to the measured low energy couplings $g_{i j}=4 \pi a_{i j} / \mathrm{m}$, where $a_{i j}$ is the scattering length, by $g_{i j}^{-1}=\bar{g}_{i j}^{-1}+\int_{0}^{\Lambda} d^{3} p /(2 \pi)^{3}\left(m / p^{2}\right)=$ $\bar{g}_{i j}^{-1}+m \Lambda_{i j} / 2 \pi^{2}$, as one sees by solving the usual tmatrix equation. Thus $\bar{g}_{i j}=4 \pi /\left(m\left(a_{i j}^{-1}+r_{0, i j}^{-1}\right)\right)$, where $r_{0, i j}=\pi / 2 \Lambda_{i j}$. Physically we expect $r_{0, i j}$ to be of order the characteristic length $\left(C_{6} \mathrm{~m} / \mathrm{m}_{e}\right)^{1 / 4} a_{0}$ for the interatomic potential, where $C_{6}$ is the strength of the van der Waals interaction; in ${ }^{6} \mathrm{Li}, r_{0} \simeq 63 a_{0}$. For short range contact interactions, Eq. (8) becomes

$$
\Omega_{\mathrm{c}}=\frac{1}{n_{2}}\left(\bar{g}_{13}-\bar{g}_{12}\right)\left\langle\psi_{1}^{\dagger}(0) \psi_{2}^{\dagger}(0) \psi_{2}(0) \psi_{1}(0)\right\rangle .
$$

The correlation function is related to the free energy, $F$, of the system by the thermodynamic identity

$$
\frac{\partial(F / V)}{\partial \bar{g}_{12}}=\frac{1}{V}\left\langle\partial H / \partial \bar{g}_{12}\right\rangle=\left\langle\psi_{1}^{\dagger}(0) \psi_{2}^{\dagger}(0) \psi_{2}(0) \psi_{1}(0)\right\rangle .
$$

We now take $r_{0}$ to be independent of the states of the atoms. From Eq. (9) we then find the mean shift:

$$
\Omega_{\mathrm{c}}=\left(\frac{\bar{a}_{13}}{\bar{a}_{12}}\right)\left(\frac{1}{g_{13}}-\frac{1}{g_{12}}\right) \frac{1}{n_{2}} \frac{\partial(F / V)}{\partial g_{12}^{-1}} .
$$

When the magnitude of the scattering lengths are $\gg r_{0}$, which for ${ }^{6} \mathrm{Li}$ is always valid above $B=600 \mathrm{G}$, the factor $\bar{a}_{13} / \bar{a}_{12}$ is $\simeq 1$; the shift then involves only the renormalized interactions, depending inversely on the scattering lengths. This result has the same qualitative behavior, in the regime when $k|a| \gg 1$, where $k$ is a typical particle momentum, as predictions based on a mean-field shift calculated from the real part of the forward scattering amplitude in a dilute gas in the absence of effective range contributions [5].

The derivative $\partial(F / V) / \partial g_{12}^{-1}<0$ behaves perfectly smoothly at the resonance in the 12-channel, and at zero temperature its value there is $\sim-\epsilon_{\mathrm{F}}^{2}$, where $\epsilon_{\mathrm{F}}=p_{\mathrm{F}}^{2} / 2 m$ is the free particle Fermi energy and $p_{\mathrm{F}}$ is the Fermi momentum. (At characteristic densities $\sim 2 \times 10^{13} \mathrm{~cm}^{-3}$, $p_{\mathrm{F}}^{-1} \sim 2000 a_{0}[5]$.) $\quad$ We assume that $n_{1}=n_{2}=n$ from here on. From the Monte Carlo calculations in Ref. 8] of the energy $E$ at zero temperature we estimate that $\partial(E / V) / \partial g_{12}^{-1} \simeq-0.50 \epsilon_{\mathrm{F}}^{2}$ at the 12-resonance at $B \simeq 834 \mathrm{G}$. Then $\Omega_{\mathrm{c}}=g_{13}^{-1} \partial(F / V) / \partial g_{12}^{-1} \sim-p_{\mathrm{F}} / m a_{13}$; this result, valid for $a_{13} \gg r_{0}$, scales as $\sqrt{\epsilon_{F}}$. On the other hand, at a 13-resonance $(B \simeq 690 \mathrm{G}), \Omega_{\mathrm{c}}=$ $-1 /\left(n g_{12}\right) \partial(F / V) / \partial g_{12}^{-1}$. As we see, the average clock shift remains finite, even when scattering is resonant.

Figure 1 shows the correlation function $\partial(E / V) / \partial a_{12}^{-1}$ calculated from the data of Ref. [8] as a function of $B$ for equal populations at zero temperature. In the BCS limit, the correlation function tends to the Hartree-BCS result, $n^{2}+\Delta^{2} / \bar{g}_{12}^{2}$, where $\Delta$ is the gap, while in the BEC limit $\left(p_{\mathrm{F}} a_{12} \ll 1\right)$ the system is composed entirely of 12 -molecules with binding energy $1 / m a_{12}^{2}$, and $\partial E / \partial g_{12}^{-1}=-4 \pi n / m^{2} a_{12}$, diverging as $a_{12} \rightarrow 0$. For small positive scattering length in a gaseous atomic state limit the correlation function tends to the Hartree value $n^{2}$, as on the BCS side. The large drop in $\partial E / \partial a_{12}^{-1}$ seen in Fig. 1 for $B$ below $\sim 700 \mathrm{G}$ reflects the presence of 12 -molecules in the initial state.

In the limit in which the bare couplings are small, $|\bar{a}| \ll$ $r_{0}$, the bare and renormalized couplings are equal, and the correlation function becomes simply $n^{2}$; thus

$$
\Omega_{\mathrm{c}}=n\left(g_{13}-g_{12}\right),
$$

the expected weak coupling result. The intermediate regime in which $r_{0} \ll|a| \ll p_{\mathrm{F}}^{-1}$ is more complicated 


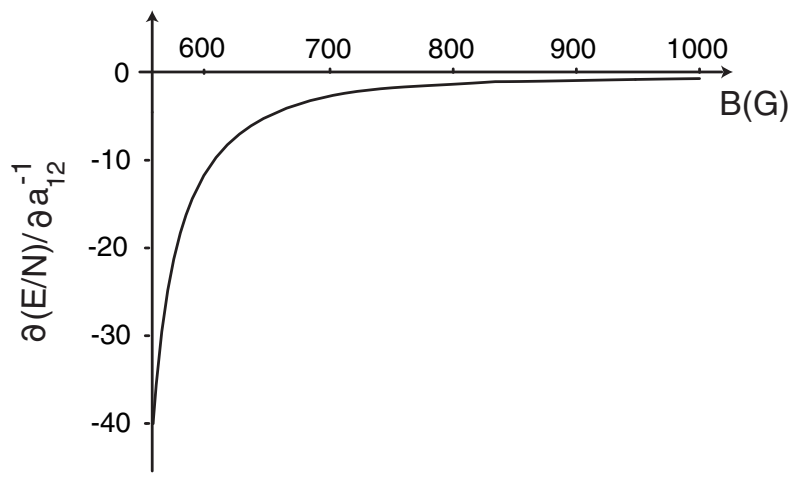

FIG. 1: Correlation function $\partial(E / N) / \partial a_{12}^{-1}$ in units of $p_{\mathrm{F}} / 2 m$ calculated from the data of Ref. [8].

since here short range correlations as well as bound states in the appropriate channel play a role. From Eq. (11) we find in this regime for an initial state without 12molecules that

$\Omega_{\mathrm{c}}=\left(g_{12}-\frac{g_{12}^{2}}{g_{13}}\right) n=\left[\left(g_{13}-g_{12}\right)-\frac{\left(g_{13}-g_{12}\right)^{2}}{g_{13}}\right] n$

which differs from the weak coupling result (12) by a factor $g_{12} / g_{13}$. To derive this result, we note that the correlation function in the absence of molecules is given by the Hartree approximation, $n^{2}$, multiplied by the square of the Jastrow factor that takes into account short-range correlations due to the interatomic interaction. At low energy, the Jastrow factor at the range of the forces is essentially $1-a_{12} / r_{0}=g_{12} / \bar{g}_{12}$, the ratio of the exact to the non-interacting two particle wave function. Thus $\left\langle\psi_{1}^{\dagger}(0) \psi_{2}^{\dagger}(0) \psi_{2}(0) \psi_{1}(0)\right\rangle=\left(g_{12} / \bar{g}_{12}\right)^{2} n^{2}[11]$.

The difference between the result (13) and Eq. (12) lies in the distribution of spectral weight for transitions in the two cases. The first term $g_{13}-g_{12}$ in the last expression in Eq. (13) is the weak coupling result.

The second term however arises from short range correlations, including, for $g_{13}>0$, 13-molecules. The squared matrix element for free-bound transitions is proportional to $\left(g_{13}-g_{12}\right)^{2}$ [9], and the $1 / g_{13}$ dependence reflects the fact that the binding energy of the molecule $\left(1 / m a_{13}^{2}\right)$ increases with decreasing $g_{13}$. (Strongly bound 13-molecular states are not described by the simple contact interaction model, but frequencies of transitions to them in general lie outside the range explored in experiment.) For $a_{13}<0$, there are no bound states, but higher-lying states with kinetic energies of order $1 / m a_{13}^{2}$, arising from short-range correlations, extend the spectral weight out to frequencies of order $\sim 1 / m a_{13}^{2}$, and contribute $\sim-1 / a_{13}$ to the sum rule [12].

In the BEC limit, $p_{\mathrm{F}} a_{12} \ll 1$ the system is composed entirely of molecules of binding energy $E_{B}=$ $1 / m a_{12}^{2}$. The energy density of the system is just $E / V=$ $-n / m a_{12}^{2}$, and from Eq. (11) we obtain

$$
\Omega_{c}=2 E_{B}\left(1-a_{12} / a_{13}\right)
$$

as can also be calculated from Ref. [9] .

In applying our results to experiment it is important to consider the extent to which, for $a_{12}>0$, molecules are present in the initial state. The experiment of Ref. [5] is carried out on a timescale short compared with that to create diatomic molecules via three-body collisions. Thus the correlation function entering the sum rule is that of the molecule-free metastable state. On the other hand, in the initial state in the experiment of Ref. 3] molecular states were equilibrated, and their effects must be included in the correlation function. Furthermore comparison with experiment requires that measurements be carried out over the entire frequency range, on scales $\sim 1 / m a_{13}^{2}$, where there are significant contributions to the spectral weight.

The solid line (a) and (b) in Fig. 2 shows the predicted clock shift for equilibrated ${ }^{6} \mathrm{Li}$, in units of $\epsilon_{\mathrm{F}} / \hbar$ in the region $\sim 600-1200 \mathrm{G}$ at zero temperature. Scattering lengths are taken from Ref. [14], and we assume $p_{\mathrm{F}}=$ $1 / 2000 a_{0}^{-1}$. The dashed-dot line (c) shows the result (14) for a non-interacting gas of molecules. The dashed part of curve (a) below the 12-resonance, the difference of the full Monte-Carlo result (b) in the 12-molecular region, and the two-body contribution (c), is a measure of the manybody contribution to the shift below the 12-resonance.

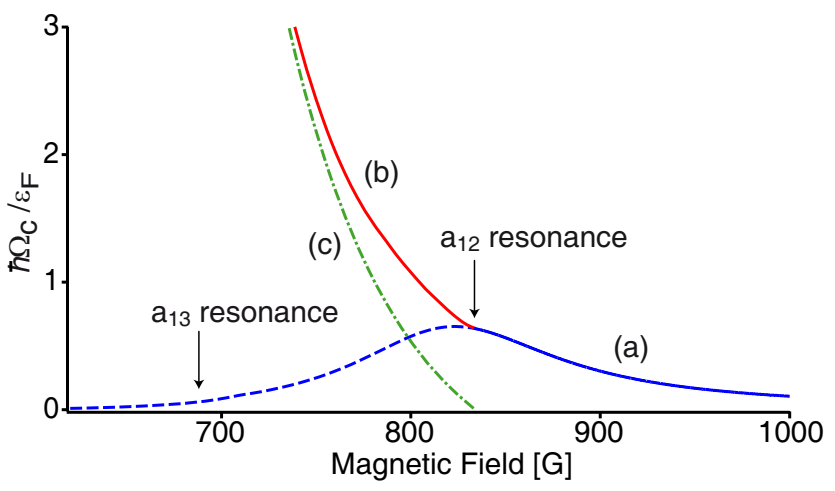

FIG. 2: Calculated clock shift for a gas of ${ }^{6} \mathrm{Li}$ atoms at zero temperature as a function of magnetic field. See text for details.

The correct picture of rf field excitation combines both coherent rotation of the initial state with transitions that change the number of elementary excitations of the system and thus lead to a width, $\Gamma$, of the spectrum $I(\omega)$. For times short compared to $\omega_{\mathrm{R}}^{-1}$ and $\Gamma^{-1}$ the rf field indeed starts to rotate all $|2\rangle$ atoms coherently into $|3\rangle$. For a strong drive, with $\omega_{\mathrm{R}} \gg \Gamma$, the many-body state undergoes Rabi oscillations, damped on a time scale $\sim \Gamma^{-1}$. The long-time behavior, when all oscillations have damped out, is captured by the Golden Rule. 
Under an instantaneous rotation of $|2\rangle$ to $|\beta\rangle$, the $|1 \beta\rangle$ state produced has the same spatial wave function as $|12\rangle$. However the bare amplitude for $1, \beta \rightarrow 1, \beta$ scattering, $\bar{g}_{1 \beta}=\cos ^{2} \theta \bar{g}_{12}+\sin ^{2} \theta \bar{g}_{13}$, differs from the bare 12 amplitude by $\delta \bar{g}=\bar{g}_{1 \beta}-\bar{g}_{12}=\sin ^{2} \theta\left(\bar{g}_{13}-\bar{g}_{12}\right)$. One can understand Eq. (9) from this point of view, by noting that since the spatial wave functions of $|1 \beta\rangle$ and $|12\rangle$ are identical, the difference of their energies (and free energies at finite temperature) is, for small $\theta$, $E_{1 \beta}-E_{12}=\delta \bar{g}\left\langle\psi_{1}^{\dagger}(0) \psi_{2}^{\dagger}(0) \psi_{2}(0) \psi_{1}(0)\right\rangle$.

The rotated state $|1 \beta\rangle$ is not in fact an energy eigenstate, and thus the response has a width. Under rotation the interaction energy becomes

$$
\begin{array}{r}
H_{\mathrm{int}}=\left[\bar{g}_{12} \cos ^{2} \theta+\bar{g}_{13} \sin ^{2} \theta\right] \psi_{1}^{\dagger} \psi_{\beta}^{\dagger} \psi_{\beta} \psi_{1} \\
+\left[\bar{g}_{13} \cos ^{2} \theta+\bar{g}_{12} \sin ^{2} \theta\right] \psi_{1}^{\dagger} \psi_{\alpha}^{\dagger} \psi_{\alpha} \psi_{1} \\
+\left(\bar{g}_{13}-\bar{g}_{12}\right) \sin \theta \cos \theta \psi_{1}^{\dagger}\left(\psi_{\alpha}^{\dagger} \psi_{\beta}+\psi_{\beta}^{\dagger} \psi_{\alpha}\right) \psi_{1},
\end{array}
$$

where $|\alpha\rangle=\cos \theta|3\rangle-\sin \theta|2\rangle$ is the superposition of single particle states $|2\rangle$ and $|3\rangle$ orthogonal to $|\beta\rangle$. The first term in Eq. (15) is the original interaction with a modified coupling constant, in terms of states $|1\rangle$ and $|\beta\rangle$, instead of $|1\rangle$ and $|2\rangle$. The second term acting on a rotated state without $\alpha$ atoms present vanishes. The final term, however, mixes the two particles in states $|1\rangle$ and $|\beta\rangle$ into $|1\rangle$ and $|\alpha\rangle$. Thus while the external rf field coherently rotates the initial state, the latter interaction decoheres the rotated state, leading to a mixture of energy eigenstates and a response with nonzero width. Even in the absence of the final term, the rotated state is not an eigenstate of $H_{\mathrm{s}}$, since the spatial wave function of an eigenstate with $\bar{g}_{1 \beta}$ differs from that with the original $\bar{g}_{12}$.

The lowest order perturbative approach does not include collective effects, e.g., screening of the interaction by the medium, and fails to satisfy the sum rule in the presence of interactions. As indicated in Ref. [7], these effects must be included by summing chains of bubble diagrams. Such terms are especially important in the superfluid state, since they take into account transitions to states in which the phase of the condensate is altered globally.

In summary, we have shown from sum-rule arguments that the average clock shifts in strongly interacting Fermi gases are finite at Feshbach resonances. The interaction model we have employed is admittedly crude, since it does not allow for the many bound states that exist for real interatomic potentials. Detailed calculations of the spectral distribution must be done microscopically. In addition to the self-energy corrections to propagators, it is necessary to include vertex corrections, as pointed out in Ref. 7]. Such effects are important even in a low density gas, where one finds interference terms between 12- scattering and 13-scattering processes [13]. These processes, which correspond to the Aslamazov-Larkin diagrams for fluctuation-induced effects in superconductors 15], will be discussed in a future publication. In addition, for detailed comparison with experiment it is necessary to take into account the inhomogeneity of the particle density.

This research was supported in part by NSF Grants PHY03-55014, PHY05-00914, and PHY07-01611. We are grateful for the hospitality of the $\mathrm{ECT}^{*}$ in Trento (Italy) which enabled this collaboration, and thank E. Demler, S. Giorgini, W. Ketterle, W. Phillips, W. Zwerger, and especially H.T.C. Stoof, for helpful input.

[1] M. W. Zwierlein, Z. Hadzibabic, S. Gupta and W. Ketterle, Phys. Rev. Lett. 91, 250404 (2003).

[2] C. A. Regal, C. Ticknor, J. L. Bohn, and D. S. Jin, Nature 424, 47 (2003).

[3] C. Chin, M. Bartenstein, A. Altmeyer, S. Riedl, S. Jochim, J. H. Denschlag and R. Grimm, Science 305, 1128 (2004).

[4] C. H. Schunck, Y. Shin, A. Schirotzek, M. W. Zwierlein, and W. Ketterle, Science 316, 867 (2007).

[5] S. Gupta, Z. Hadzibabic, M. W. Zwierlein, C. A. Stan, K. Dieckmann, C. H. Schunck, E. G. M. van Kempen, B. J. Verhaar, and W. Ketterle, Science 300, 1723 (2003).

[6] J. Kinnunen, M. Rodríguez and P. Törmä, Science 305, 1131 ( 2004).

[7] Z. Yu and G. Baym, Phys. Rev. A 73, 063601 (2006).

[8] G. E. Astrakharchik, J. Boronat, J. Casulleras, and S. Giorgini, Phys. Rev. Lett. 93, 200404 (2004).

[9] C. Chin and P. Julienne, Phys. Rev. A 71, 012713 (2005).

[10] G. M. Bruun and G. Baym, Phys. Rev. A 74, 033623 (2006).

[11] Integrating $\partial(E / V) / \partial g_{12}^{-1}=-g_{12}^{2} n^{2}$ with respect to $\bar{g}$ we recover immediately the low-energy result, $E_{\text {int }} / V=$ $g_{12} n^{2}$.

[12] One can calculate the total weight at frequencies $\lesssim \epsilon_{\mathrm{F}}$ energy using an effective low-energy Hamiltonian with renormalized couplings. The correlation function for the low energy excitations of the system is given by the Hartree approximation, and so the average shift of transitions with energies $\lesssim \epsilon_{\mathrm{F}}$ is again given by Eq. (12). For an application of sum rules to weakly interacting gases using a low energy effective interaction, see M. Ö. Oktel, T. C. Killian, D. Kleppner, and L. S. Levitov, Phys. Rev. A 65, 033617 (2002).

[13] J. M. V. A. Koelman, S. B. Crampton, H. T. C. Stoof, O. J. Luiten, and B. J. Verhaar, Phys. Rev. A 38, 3535 (1988).

[14] M. Bartenstein, A. Altmeyer, S. Riedl, R. Geursen, S. Jochim, C. Chin, J. H. Denschlag, R. Grimm, A. Simoni, E. Tiesinga, C. J. Williams, and P. S. Julienne, Phys. Rev. Lett. 94, 103201 (2005).

[15] L. G. Aslamazov and A. I. Larkin, Phys. Lett. A 26, 228 (1968). 